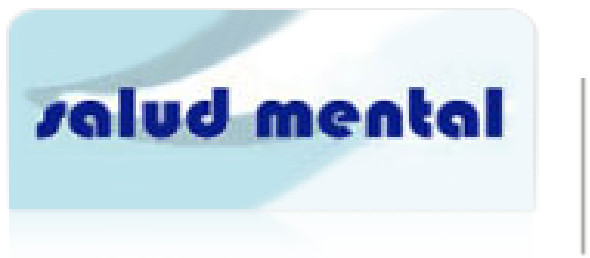

Salud Mental

ISSN: 0185-3325

perezrh@imp.edu.mx

Instituto Nacional de Psiquiatría Ramón

de la Fuente Muñiz

México

Gómez-Chavarín, Margarita; Díaz-Pérez, Rosalinda; Morales-Espinosa, Rosario; Fernández-Ruiz, Juan; Roldán-Roldán, Gabriel; Torner, Carlos

Efecto de la exposición al pesticida rotenona sobre el desarrollo del sistema dopaminérgico nigroestriatal en ratas

Salud Mental, vol. 36, núm. 1, enero-febrero, 2013, pp. 1-8

Instituto Nacional de Psiquiatría Ramón de la Fuente Muñiz

Distrito Federal, México

Disponible en: http://www.redalyc.org/articulo.oa?id=58225671001

- Cómo citar el artículo

- Número completo

- Más información del artículo

- Página de la revista en redalyc.org

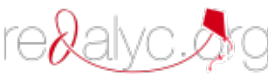

Sistema de Información Científica

Red de Revistas Científicas de América Latina, el Caribe, España y Portugal

Proyecto académico sin fines de lucro, desarrollado bajo la iniciativa de acceso abierto 


\title{
Efecto de la exposición al pesticida rotenona sobre el desarrollo del sistema dopaminérgico nigro-estriatal en ratas
}

\author{
Margarita Gómez-Chavarín,' Rosalinda Díaz-Pérez, ${ }^{1}$ Rosario Morales-Espinosa, ' Juan Fernández-Ruiz, ${ }^{1}$ \\ Gabriel Roldán-Roldán, ${ }^{1}$ Carlos Torner ${ }^{2}$
}

Artículo original

\section{SUMMARY}

Rotenone is a pesticide used in Mexico, despite the experimental evidence showing dopaminergic neurons degeneration induced by this compound, which may lead to a psychomotor impairment. However, the possible effects of rotenone on the offspring when they are indirectly exposed through their mothers are still unknown. In this study rotenone was administered to female rats during pregnancy and nursing, in order to assess its effects on the offspring's dopaminergic neurons in the substantia nigra, as well as on motor coordination at 30 or 60 postnatal days.

Six groups of pregnant Wistar rats were used: an intact control group, a vehicle group injected with the rotenone solvent, and four groups injected subcutaneously with the following doses of rotenone: $0.2,0.4,0.6$, and $1 \mathrm{mg} / \mathrm{kg} /$ day. In a parallel experiment, the offspring of other groups of dams treated with rotenone $1 \mathrm{mg} / \mathrm{kg} /$ day, or controls vehicle-treated, were used to evaluate motor coordination at 30 and 60 postnatal days.

Rotenone treated dams showed a significant lower amount of dopaminergic neurons in the substantia nigra, but only with the $1 \mathrm{mg} / \mathrm{kg}$ dose. This effect was also observed in the offspring but at all doses of rotenone tested, either at 30 or 60 postnatal days. Furthermore, the offspring of rotenone exposed dams significantly increased the time in which they accomplished the motor coordination test, compared to the offspring of control dams.

These data indicate that rotenone is able to damage the dopaminergic neurons of the offspring though their mothers. This effect requires lower rotenone doses than in adult rats. The reduced number of dopaminergic neurons at early stages of life enhances the risk of developing disorders related to the brains' dopaminergic system.

Key words: Rotenone, Parkinson disease, tyrosine hydroxilase, substantia nigra, attention deficit hyperactivity disorder, and motor coordination.

\section{RESUMEN}

La rotenona es un pesticida utilizado en México a pesar de que se ha demostrado experimentalmente que produce una degeneración de las neuronas dopaminérgicas, y puede derivar en deterioro psicomotor. Sin embargo, no existen estudios de la exposición indirecta a rotenona a través de las madres en el efecto que produzca sobre su descendencia. Nosotros administramos rotenona a ratas durante la gestación y la lactancia para evaluar las alteraciones producidas sobre las neuronas dopaminérgicas y la coordinación motora de sus crías, a los $30 \circ 60$ días posnatales. Para cuantificar las neuronas inmunorreactivas a tirosina hidroxilasa de la sustancia nigra, se inyectaron subcutáneamente seis grupos de hembras Wistar: intactas (control), con solvente de rotenona (vehículo) y cuatro grupos con rotenona en dosis: $0.2,0.4,0.6$ y $1.0 \mathrm{mg} / \mathrm{kg} /$ día. En un experimento paralelo, las crías de otros grupos de hembras tratadas con rotenona $1 \mathrm{mg} / \mathrm{kg} /$ día o controles fueron evaluados en la prueba de coordinación motora a los 30 y 60 días posnatales.

Las madres tratadas con $1 \mathrm{mg} / \mathrm{kg}$ de rotenona tuvieron menos neuronas dopaminérgicas en la sustancia nigra. Dicho efecto se observó también en las crías, pero con todas las dosis de rotenona utilizadas, tanto a los 30 como a los 60 días posnatales. Además, la exposición indirecta a rotenona aumentó significativamente el tiempo que requirieron las crías para ejecutar la prueba de coordinación motora.

Estos datos indican que la rotenona es capaz de inducir daño en las neuronas dopaminérgicas de las crías cuando son expuestas a través de sus madres. Este efecto en las crías se observa con dosis menores de rotenona que en ratas adultas. Por lo tanto, los individuos indirectamente expuestos a rotenona podrían tener menos neuronas dopaminérgicas desde etapas tempranas de la vida, lo que aumenta el riesgo de desarrollar trastornos relacionados con el sistema dopaminérgico.

Palabras clave: Rotenona, enfermedad de Parkinson, trastorno por déficit de atención con hiperactividad, sustancia nigra, tirosina hidroxilasa, coordinación motora.

Facultad de Medicina, Universidad Nacional Autónoma de México, México.

2 División de Ciencias Biológicas y de la Salud. Universidad Autónoma Metropolitana, México.

Correspondencia: Carlos A. Torner Aguilar. División Ciencias Biológicas y de la Salud, Universidad Autónoma Metropolitana-Xochimilco. Calz. del Hueso 1 100, Villa Quietud, Coyoacán, 04960, México, DF. Tel. 54837000 ext. 3260. Fax: 5483 7200. E-mail: ctorner@correo.vam.xoc.mx

Recibido primera versión: 10 de octubre de 2011. Segunda versión: 28 de marzo de 2012. Tercera versión: 3 de mayo de 2012. Aceptado: 14 de mayo de 2012. 


\section{INTRODUCCIÓN}

La rotenona (ROT) es un compuesto que se extrae de las plantas del género Lonchocarpus, que actúa como pesticida. Por su origen natural, la ROT es ampliamente utilizada como insecticida en el control de plagas en múltiples cultivos, y como piscicida para el control de ciertas especies de peces no deseables en mantos acuíferos. Su alta liposolubilidad le permite atravesar fácilmente las membranas biológicas. Dentro de las células, la ROT tiene un efecto inhibitorio altamente específico sobre el complejo I de la cadena respiratoria mitocondrial. ${ }^{1-3}$ En México se usa particularmente en la producción camaronera de las costas del Pacífico, donde los estanque son tratados con ROT al 5.8\%, sin que existan estudios del impacto que ésta pueda tener en la salud humana por exposición ocupacional o por consumo. Dhillon et al. ${ }^{3}$ analizaron el efecto de la exposición ocupacional a ROT en una población de Texas y encontraron un incremento en la incidencia de enfermedad de Parkinson asociado con el uso de este pesticida; sin embargo, en México el panorama es aún desconocido.

La evidencia actual apunta a que la exposición a pesticidas como ROT, maneb o paraquat, produce lesiones degenerativas en las neuronas dopaminérgicas cerebrales. ${ }^{4-6}$ La disminución de este tipo de neuronas tiene una mayor probabilidad de producir alteraciones psicomotoras en un síndrome tipo enfermedad de Parkinson; sin embargo, a la fecha se ha estudiado escasamente el efecto de la degeneración de células dopaminérgicas sobre alteraciones funcionales que deriven en trastornos mentales. Se ha encontrado que la administración subcutánea de ROT en ratas adultas resulta en la degeneración del sistema dopaminérgico nigroestriatal, lo que produce deterioro psicomotor, además de favorecer la agregación de la proteína $\alpha$-sinucleína en las terminales sinápticas de dicha vía. ${ }^{1,7} \mathrm{El}$ hecho de que la ROT induzca estos marcadores citológicos de la enfermedad de Parkinson en roedores ha contribuido a que se le considere como modelo animal de dicha enfermedad. Sin embargo, otros núcleos dopaminérgicos cerebrales también podrían estar afectados, aunque el estudio funcional de las alteraciones psiquiátricas que generan resulta particularmente difícil de evaluar en los animales.

En este trabajo estudiamos el efecto que tiene la exposición a ROT en etapas muy tempranas de la vida sobre las neuronas dopaminérgicas de la sustancia nigra (SN) de ratas. El pesticida fue administrado a través de la madre desde el inicio de la gestación hasta el término de la lactancia, lo que permitió estudiar su efecto sobre la descendencia, usando como referencia el daño provocado en las madres, puesto que éste ya se ha caracterizado en animales adultos. Las ratas gestantes fueron evaluadas al final del tratamiento de 51 días, mientras que sus crías fueron evaluadas a los 30 y 60 días posteriores al nacimiento (DPN). La evaluación consistió en contar el número de neuronas con inmunoreactividad positiva para tirosina hidroxilasa (IR-TH) en la SN, la cual marca a las células dopaminérgicas debido a que la tirosina hidroxilasa (TH) es la enzima limitante en la vía biosintética de la dopamina. La disminución de las neuronas IR-TH es un indicativo de daño sobre este núcleo dopaminérgico. Adicionalmente, evaluamos el efecto sobre la coordinación motora mediante la prueba de la viga inclinada de Drucker. ${ }^{8}$

\section{MATERIALES Y MÉTODOS}

\section{Sujetos y procedimientos}

Veinticuatro ratas Wistar hembras de $250 \mathrm{~g}$ se dividieron en doce cajas; se colocaron dos hembras con un macho sexualmente experto. Después de 48 h se verificó mediante el tapón vaginal que las hembras estuvieran preñadas, lo que determinó el inicio del tratamiento con ROT. Las hembras preñadas se colocaron en cajas individuales y se mantuvieron en condiciones estándar con un ciclo invertido de luz-obscuridad de 12-12 horas (las luces se apagaban a las 7 am), a temperatura $\left(21 \pm 1.0^{\circ} \mathrm{C}\right)$ y humedad $(55 \pm 5 \%)$ constantes y con agua y alimento ad libitum. Las ratas gestantes se dividieron en seis grupos $(\mathrm{n}=4 \mathrm{c} / \mathrm{u})$ : un grupo control intacto que no recibió ningún tratamiento; un grupo control inyectado con $100 \mu \mathrm{l}$ de una solución 1:1 de polietilenglicol y dimetilsulfóxido (PEG: DMSO) denominado grupo "vehículo" por recibir sólo el solvente de la ROT (Cat. R8875, Sigma, San Luis, MO), y cuatro grupos experimentales con diferentes dosis de ROT: $0.2 \mathrm{mg} / \mathrm{kg}, 0.4 \mathrm{mg} / \mathrm{kg}$, $0.6 \mathrm{mg} / \mathrm{kg}$ y $1.0 \mathrm{mg} / \mathrm{kg}$, respectivamente.

Tanto el vehículo como la ROT fueron administrados diariamente por vía subcutánea a las ratas gestantes durante los 21 días de gestación y los 30 días de lactancia. Todos los animales fueron manejados de acuerdo con la Norma Oficial Mexicana (NOM-062-ZOO-1999). Al final del tratamiento se extrajeron los cerebros de las madres y de las crías de 30 días posnatales (DPN). Los cerebros de las crías de 60 DPN se estudiaron con el fin de tener un grupo para evaluar el efecto de la ROT 30 días después de terminada la exposición. Los cerebros se congelaron a $-20^{\circ} \mathrm{C}$ para hacer cortes coronales de $40 \mu \mathrm{m}$, y las secciones fueron procesadas con anticuerpos contra $\mathrm{TH}$, con el fin de marcar inmunohistoquímicamente las células de la SN que tuvieran esta enzima (neuronas dopaminérgicas).

\section{Procedimiento inmunohistoquímico para TH}

Las ratas hembras y sus crías fueron anestesiadas con una sobredosis de pentobarbital sódico, 1 o $0.5 \mathrm{ml}$, respectivamente (Pfizer, México, DF, México) y se perfundieron transcardialmente con $200 \mathrm{ml}$ de una solución PBS (solución salina en buffer de fosfatos: 0,1 M, pH 7.4, NaCl $130 \mathrm{mM}$, $\mathrm{Na}_{2} \mathrm{HPO}_{4} 7 \mathrm{mM}$ y NaH $\mathrm{PO}_{4} 3 \mathrm{mM}$ ), y posteriormente fueron fijadas con $250 \mathrm{ml}$ de paraformaldehído al $4 \%$ disuelto en la 
Efecto de la exposición al pesticida rotenona sobre el desarrollo del sistema dopaminérgico nigro-estriatal

solución PBS. Los cerebros se extrajeron y se posfijaron en la misma solución durante $12 \mathrm{~h}$, y posteriormente se colocaron en una solución crioprotectora consistente en sacarosa al $30 \%$ disuelta en solución PBS a $4^{\circ} \mathrm{C}$.

De la porción del cerebro correspondiente a la $\mathrm{SN}$, de acuerdo con el atlas estereotáxico del cerebro de la rata, ${ }^{9}$ se obtuvieron cortes de $40 \mu \mathrm{m}$ de grosor en un micrótomo de congelación (Cryo-Cut American Optical).

Para la inmunohistoquímica de $\mathrm{TH}$, las rebanadas de cerebro se colocaron en libre flotación en cajas multipozos y se lavaron tres veces con PBS durante 10 min cada lavada (PBS 3X10 min); se incubaron por una hora a $4^{\circ} \mathrm{C}$ en una solución PBS con 3\% de Triton X-100 (Sigma) y 0.01\% de albúmina bovina (BSA; Sigma). Posteriormente, las secciones fueron incubadas 12 horas en una solución de PBS que contenía el anticuerpo policlonal de conejo contra TH (1:1000; Biotecnología Santa Cruz); esta incubación se mantuvo en condiciones de agitación leve y a $4^{\circ} \mathrm{C}$. Al día siguiente, después de lavarse en PBS 3X10 min, los cortes fueron incubados en solución PBS con un anticuerpo secundario biotinilado (IgG de conejo biotinilado diluido a 1:250; ABC Vector Burlingame, CA, EE.UU.) durante $2 \mathrm{~h}$ a temperatura ambiente; al término de este tiempo los cortes fueron lavados con PBS 3X10 min. Para visualizar la reacción de la unión antígeno-anticuerpo, las secciones se incubaron $2 \mathrm{~h}$ en una solución de avidina conjugada con peroxidasa de rábano (ABC VectaStain Elite Kit Vector, Burlingame, CA, EE.UU.). Después de lavarse 3X10 min con una solución Tris- $\mathrm{HCl} 0.1$ $\mathrm{M}$, pH 7.4, las secciones fueron incubadas $5 \mathrm{~min}$ con $0.1 \%$ de diaminobencidina (Sigma), y luego se agregó peróxido de hidrogeno $\left(\mathrm{H}_{2} \mathrm{O}_{2}\right)$ al $0.24 \%$ para revelar la localización de los anticuerpos. La reacción fue monitoreada con un microscopio, y cuando era claramente visible se detuvo mediante lavados con PBS 3X5 min. Finalmente, las secciones reveladas fueron colocadas sobre portaobjetos, se deshidrataron con alcoholes y xilol, para cubrirse por último con Cytoseal XYL (Richard Allan-científico) y un cubreobjetos.

\section{Análisis inmunohistoquímico}

Para cuantificar a las neuronas IR-TH, los límites de la SN se establecieron de acuerdo con el atlas estereotáxico del cerebro de rata, ${ }^{9}$ iniciando en $-5,20$ y terminando a $-6,30 \mathrm{~mm}$ de Bregma (aproximadamente $800 \mu \mathrm{m}$ de la SN fueron evaluadas). Las imágenes fueron capturadas por una cámara (Evolution VF Cooled Color Camera Medica Cybernetics) adaptada a un microscopio (Olympus IX-71, Japón) con un aumento 4X y 20X; una vez adquiridas se analizaron con el programa Image ProPlus 6.0. Las neuronas IR-TH fueron contadas manualmente. El criterio para considerar a una neurona dopaminérgica IR-TH fue encontrar el citoplasma completamente marcado, y el núcleo perfectamente definido y sin marca. Veinte cortes del cerebro de cada animal se analizaron por dos investigadores independientes. Con es- tos datos se calculó el promedio de las neuronas IR-TH para cada uno de los 10 animales; los resultados son el promedio de las neuronas IR-TH de 10 sujetos.

\section{Prueba de coordinación motora}

Treinta críos de 30 DPN divididos en tres grupos: control, vehículo y ROT $1 \mathrm{mg} / \mathrm{kg}(\mathrm{n}=10 \mathrm{c} / \mathrm{u})$ fueron evaluados a los 30 y 60 DPN con la prueba de coordinación motora diseñada y validada por Drucker y García. ${ }^{8}$ Este dispositivo consiste en una viga de madera de $2 \mathrm{~m}$ de largo, colocada con una inclinación de $15^{\circ}$, para que la rata la escale. En el extremo superior de la viga se coloca su caja-hogar como estímulo para que ejecute la prueba. Inicialmente, las ratas se entrenan a trepar sobre una viga de $24 \mathrm{~mm}$ de ancho durante cinco días antes de realizar la prueba; la prueba se inicia cambiando al azar el grosor de la viga en anchos de 9, 6 y $3 \mathrm{~mm}$. La rata se coloca en la parte baja de la viga y se le permite caminar hasta alcanzar el extremo superior registrando el tiempo en el que llega. Se emplea un limite máximo de 120 seg, al término del cual si la rata no ha alcanzado su caja-hogar se remueve manualmente y coloca en su caja y recibe una calificación de $120 \mathrm{seg}$. Los resultados se expresaron como el promedio del tiempo total (seg) que tardaron los 10 sujetos en cada condición experimental para ejecutar la prueba.

\section{RESULTADOS}

\section{Efecto de la ROT en las ratas gestantes}

Las ratas gestantes tratadas con ROT durante 51 días tuvieron un menor número de neuronas IR-TH en la SN. La figura 1 (paneles A, D, J, M y P) muestra imágenes representativas de las áreas correspondientes a la $\mathrm{SN}$, donde se observa una disminución de las neuronas IR-TH proporcional al aumento de la dosis de ROT. La media del número de neuronas IR-TH en las hembras adultas sin tratamiento (control) fue 200.5, y en las tratadas con el vehículo fue 160.5; los animales tratados con ROT tuvieron valores de 219.5, 171.5, 128.5 y 41.75 neuronas por campo, que corresponden a las dosis de $0.2,0.4,0.6$ y $1.0 \mathrm{mg} / \mathrm{kg}$ de ROT, respectivamente (figura 2).

$\mathrm{El}$ análisis de varianza (ANOVA) de una vía reveló un efecto significativo del tratamiento $\left(\mathrm{F}_{(5,18)}=11.04 ; \mathrm{p}<0.0001\right)$. La prueba post hoc de comparaciones múltiples de Tukey mostró que los grupos control, vehículo y con $0.2 \mathrm{mg} / \mathrm{kg}$ de ROT tuvieron diferencias significativas contra el grupo que recibió la mayor dosis de ROT $(\mathrm{p}<0.001)$.

\section{Efecto de la ROT en las crías}

El número de neuronas IR-TH en la SN de las crías mostró un patrón similar al de sus madres tratadas con ROT: las 

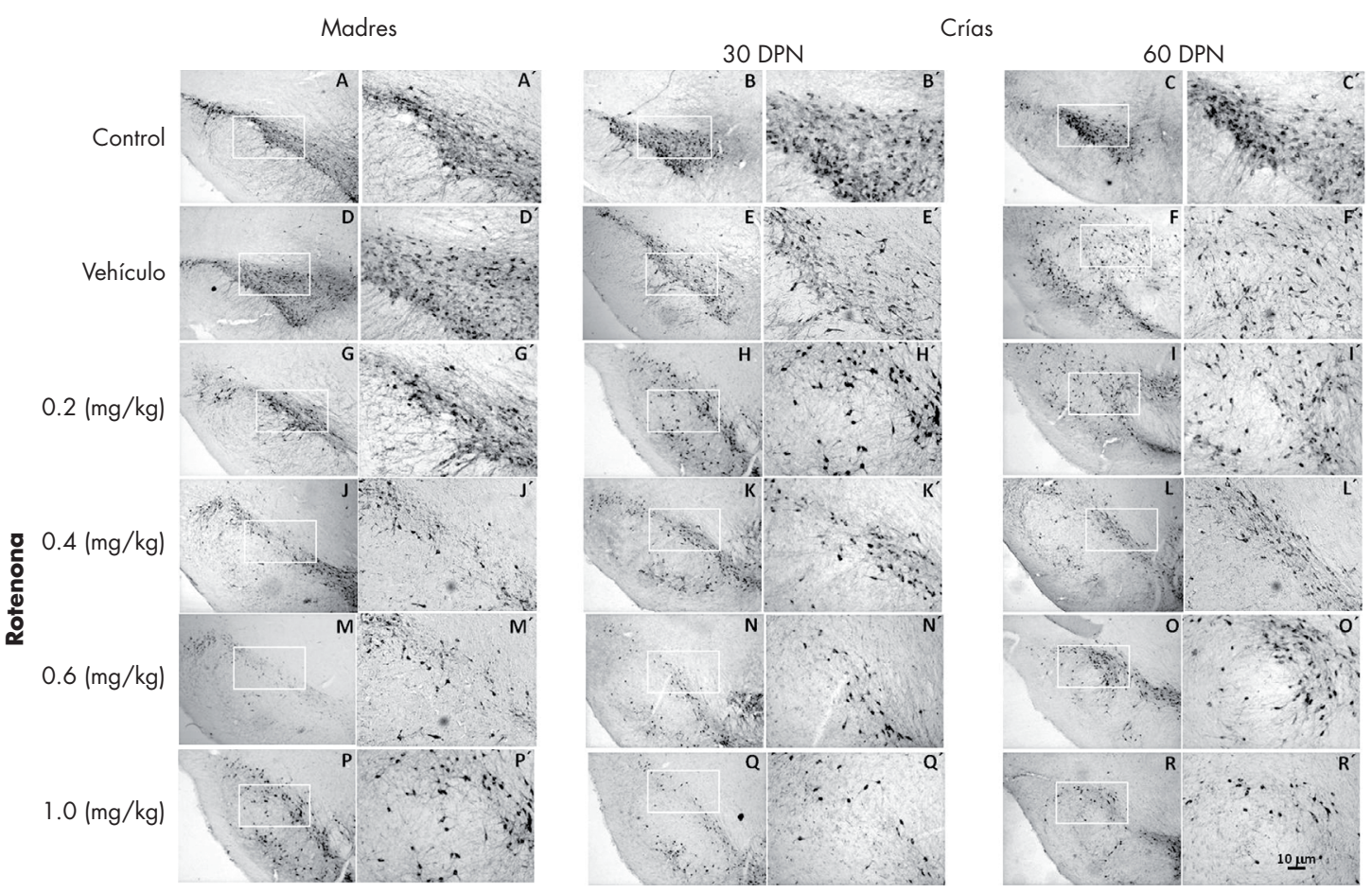

Figura 1. Fotografías representativas de la substantia nigra de las madres tratadas con diferentes dosis de ROT y sus crías. Las primeras dos columnas (letras $A, D, G, J, M$ y $P$, y las marcadas con el apostrofo ') presentan el tejido de ratas madres a un aumento $4 \mathrm{X}$ (izquierda) y $20 \mathrm{X}$ (derecha), donde se puede observar una menor densidad de neuronas IR-TH conforme se incrementa la dosis de ROT. Los paneles $\mathrm{B}, \mathrm{E}, \mathrm{H}, \mathrm{K}, \mathrm{M}$ y $\mathrm{P}$ presentan substantia nigra de las crías de 30 días posnatales con el mismo aumento (4X izquierda y $20 \mathrm{X}$ derecha). Los paneles $\mathrm{C}, \mathrm{F}, \mathrm{I}, \mathrm{L}, \mathrm{N}$ y $\mathrm{Q}$ presentan la substantia nigra en las crías de 60 días posnatales con el mismo aumento (ibid). En las fotografías de la derecha se muestra el área representativa de la substantia nigra en la que se llevó a cabo el conteo de las neuronas IR-TH. La barra muestra una escala de $10 \mu \mathrm{m}$.

neuronas IR-TH disminuyeron conforme aumentó la dosis de ROT; este efecto se encontró en las crías de 30 DPN expuestas indirectamente in utero y durante la lactancia a ROT. La figura 1 (paneles: B, E, H, K, N y Q) muestra la SN de estos animales donde se observa la disminución en la población de neuronas IR-TH. Un efecto similar de la ROT se encontró en los animales de 60 DPN, los cuales habían estado 30 días sin exposición al xenobiótico. La figura 1 (paneles: C, F, I, L, O y R) muestra el efecto de la ROT sobre las neuronas IR-TH de dichos animales.

En los animales de 30 DPN, los valores promedio del número de neuronas en condiciones control y vehículo fueron 190.7 y 161.7, mientras que en los grupos tratados con las diferentes dosis de ROT fueron de 135, 102.9, 75.18 y 56.5, respectivamente (figura 3A). El ANOVA de una vía mostró diferencias significativas del tratamiento $\left(\mathrm{F}_{(5,54)}=1268 ; \mathrm{p}<0.0001\right)$. La prueba post hoc de comparaciones múltiples de Tukey reveló que los grupos control y vehículo tuvieron diferencias con todos los grupos tratados con ROT ( $<<0.001)$. La densidad de neuronas IR-TH disminuye de modo similar en las crías de 60 DPN; los valores promedio del número de neuronas en condiciones control y vehículo fueron 191 y 165, respectivamente, y en los grupos tratados con las diferentes dosis de ROT fueron de 106.8, 65.35, 56.38 y 32.52, respectivamente (figura 3B). El ANOVA de una vía mostró diferencias significativas por el tratamiento $\left(\mathrm{F}_{(5,54)}=344,1 ; \mathrm{p}<0.0001\right)$, y la prueba post hoc de Tukey reveló diferencias entre los grupos control y vehículo, $\mathrm{y}$ todos los grupos tratados con ROT $(\mathrm{p}<0.001)$.

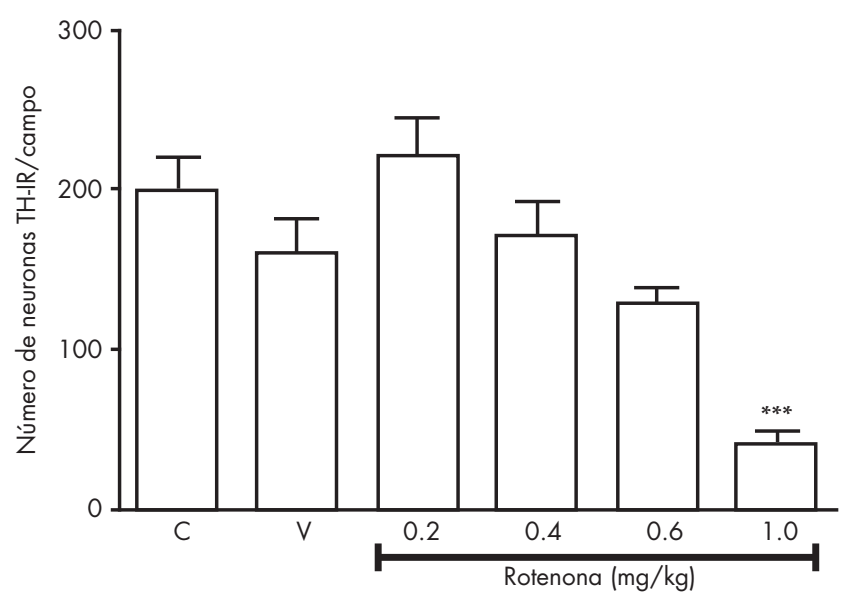

Figura 2. Número de neuronas IR-TH de la $S N$ de las ratas adultas. La gráfica muestra el efecto del tratamiento con ROT por 51 días en estas ratas. Cada barra representa el promedio \pm el error estándar del número de neuronas IR-TH por campo, obtenido a partir de 20 cortes por sujeto de cada grupo $(n=4)$. Los asteriscos $\left({ }^{* * *}\right)$ muestran al grupo con significancia estadística $(p<0.0001) . C=$ grupo control; $\mathrm{V}=$ vehículo y la barra con el rótulo Rotenona representa a los animales tratados con diferentes dosis de ésta. 

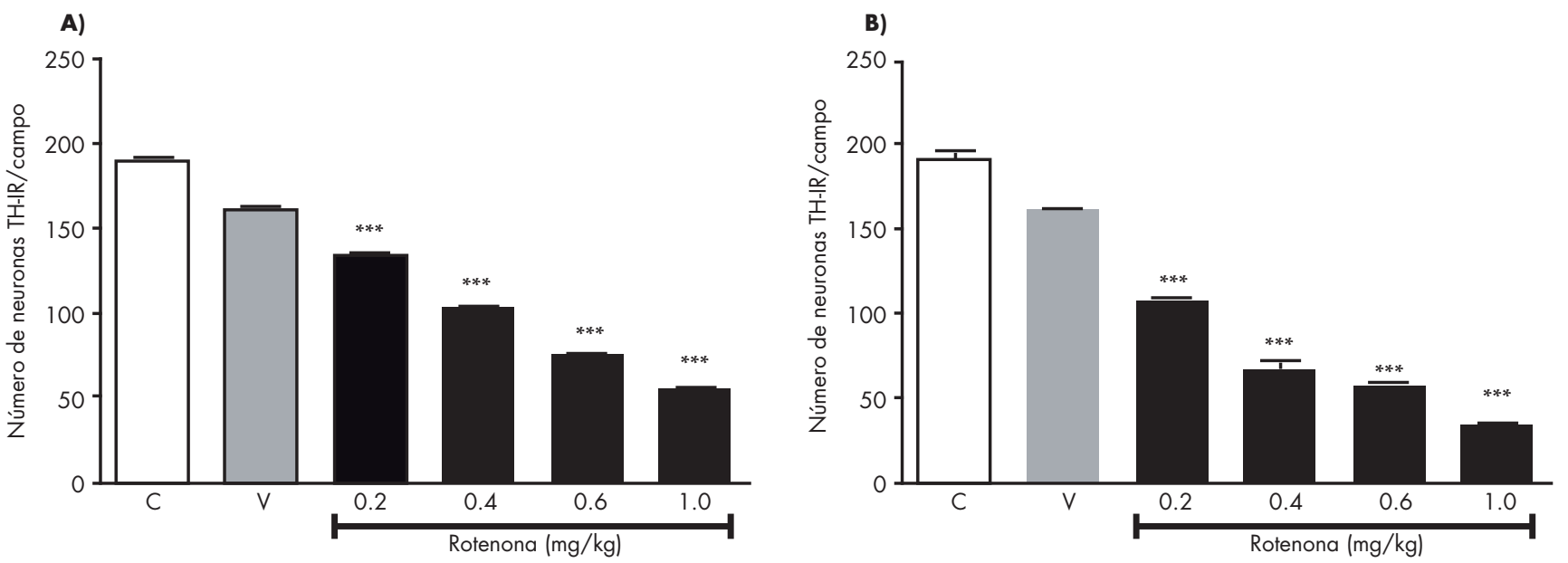

Figura 3. Número de neuronas IR-TH de la SN. Efecto de la exposición in utero y durante la lactancia a ROT en crías de 30 DPN (A) y en crías de 60 DPN (B). Cada barra representa el promedio \pm el error estándar del número de neuronas IR-TH de la substantia nigra por campo obtenidos a partir de 20 cortes por cada sujeto de cada grupo $(n=10)$. Los asteriscos ( ${ }^{* *}$ ) representan las diferencias significativas ( $<<0.0001$ ) obtenidas entre los grupos $\mathrm{C}=$ control, $\mathrm{V}=$ vehículo vs. los grupos expuestos a las diferentes dosis de ROT.

\section{Efecto de la ROT sobre la prueba de coordinación motora}

Los resultados obtenidos al evaluar a las crías a los 30 y 60 DPN en la prueba de coordinación motora se muestran en la figura 4. Las crías expuestas a $1 \mathrm{mg} / \mathrm{kg}$ de ROT requirieron más tiempo para ejecutar la prueba de coordinación motora. Como se muestra en la figura 4A, en las crías de $30 \mathrm{DPN}$ aumentó el tiempo total (seg) para ejecutar la prueba a medida que disminuía el grosor de la barra de 9, a 6 y $3 \mathrm{~mm}$. El análisis de varianza mostró un efecto significativo del tratamiento con ROT $\left(\mathrm{F}_{(8,81)}=478 ; \mathrm{p}<0.0001\right)$. La prueba post hoc de Tukey mostró que en las crías expuestas a $1 \mathrm{mg} / \mathrm{kg}$ de ROT aumentó significativamente el tiempo para ejecutar la prueba en comparación con las crías control y vehículo $(p<0.001)$. En la figura 4B se muestra que persiste el efec- to deletéreo sobre la coordinación motora en estas mismas crías a los $60 \mathrm{DPN}\left(\mathrm{F}_{(8,81)}=344.1 ; \mathrm{p}<0.0001\right)$. La prueba post hoc de Tukey mostró diferencias significativas $(\mathrm{p}<0.001)$ en el tiempo total (seg) que tardan en ejecutar la prueba bajo las mismas condiciones experimentales.

\section{DISCUSIÓN}

Existen múltiples factores ambientales que pueden provocar una variedad de efectos tóxicos en el organismo. Diversos estudios muestran que la exposición a xenobiótico como metales, ${ }^{10}$ pesticidas entre los que se incluye la ROT, ${ }^{1,4}$ fungicidas y herbicidas como el maneb y paraquat, ${ }^{11-13}$ entre varios otros, pueden generar daños en el Sistema Nervioso Central. ${ }^{14}$ Grandjean y Landrigan ${ }^{10}$ encontraron que la ex-
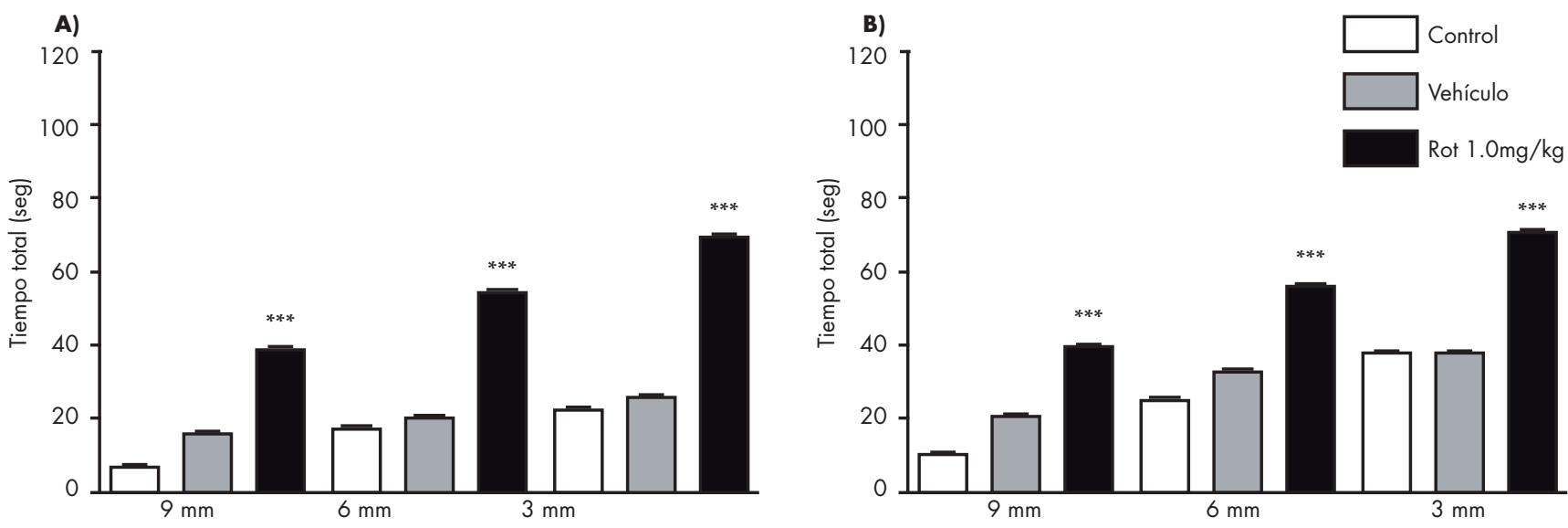

Figura 4. Tiempo empleado para ejecutar la prueba de coordinación motora. El panel A muestra los animales evaluados a los 30 DPN y el panel B las mismas crías evaluadas a los 60 DPN. Las crías expuestas a ROT (1 mg/kg) tuvieron diferencias significativas (*** $p<0.05$ ) comparada con los grupos control y vehículo. El eje de las abscisas indica el grosor de cada barra en la que los animales fueron evaluados. 
posición del feto humano a sustancias químicas industriales provocaba daños en el cerebro durante el proceso de neurodesarrollo que podían predisponer a diversas enfermedades neurológicas o psiquiátricas en el largo plazo.

Hasta donde sabemos no hay estudios previos que aborden los efectos in utero de la ROT, por lo que evaluamos en ratas Wistar la exposición a este pesticida durante la gestación y la lactancia sobre el número de neuronas dopaminérgicas de su descendencia y la coordinación motora. El diseño experimental contempló dos momentos posnatales para investigar los cambios que se manifestarán en el cerebro de las crías: uno al término de la exposición indirecta a la ROT, y el otro 30 días después de finalizar el tratamiento, para determinar si había cambios relacionados con la maduración cerebral.

La administración de ROT por vía s.c. en las madres sirvió como control del daño sobre las células dopaminérgicas, puesto que las madres y las crías estuvieron expuestas a las mismas concentraciones de ROT en sangre. Nuestros hallazgos indican que las ratas madres tuvieron una disminución significativa en el número de neuronas inmunoreactivas a $\mathrm{TH}$ (neuronas dopaminérgicas) en la $\mathrm{SN}$, pero solamente con la dosis de $1.0 \mathrm{mg} / \mathrm{kg}$. Nuestros resultados replican los hallazgos previamente reportados por Betarbet et al., ${ }^{1}$ quienes trataron ratas macho adultas con $2 \mathrm{mg} / \mathrm{kg}$ de ROT durante dos semanas y observaron una disminución en el número de neuronas IR-TH. En todos los estudios previos se han empleado dosis superiores a $2 \mathrm{mg} / \mathrm{kg}$ de ROT y se han obtenido resultados similares a los nuestros. ${ }^{1,7,15-18}$ En nuestras condiciones experimentales no observamos efectos significativos con dosis menores, por lo que proponemos que $1 \mathrm{mg} / \mathrm{kg}$ de ROT debe ser la dosis mínima efectiva para provocar un daño significativo sobre las neuronas dopaminérgicas en la SN de ratas adultas.

En un estudio previo se había sugerido que las ratas hembras son resistentes a pesticidas como maneb y paraquat; ${ }^{19}$ sin embargo, nuestros resultados no indican ninguna resistencia especial de las hembras a la ROT, lo que podría deberse a que esta última afecta vías metabólicas diferentes a las del maneb y el paraquat en su acción neurotóxica. ${ }^{20}$

El interés primordial de nuestro trabajo fue explorar los daños que la ROT puede producir sobre las neuronas dopaminérgicas de la SN durante el desarrollo de las crías. Nuestros resultados muestran que las dosis de ROT administradas a través de la madre produjeron disminución significativa en el número de neuronas dopaminérgicas (IR$\mathrm{TH})$. Esto contrasta con el efecto observado en las madres, en las cuales sólo la dosis mayor de ROT provocó un decremento significativo. Esta diferencia puede deberse a que el fenotipo neuronal está definido en las madres y esto les confiere resistencia a los efectos a las neurotoxinas, mientras en las crías el fenotipo neuronal no está comprometido y el proceso de diferenciación las hace vulnerables a daños ocasionados por factores externos. ${ }^{21}$ Además, en las crías parece que la maduración cerebral posnatal es también un factor importante para el efecto de la ROT, pues los animales de 60 DPN, los que estuvieron 30 días sin exposición, mostraron una disminución mayor en el número de neuronas IR$\mathrm{TH}$ en comparación con los animales de $30 \mathrm{DPN}$, los que habían terminado su exposición al pesticida cuando fueron evaluadas. Esto podría atribuirse a la acumulación de ROT en el tejido adiposo, que pudiera liberarse de manera tónica posteriormente en las crías.

Aunque ambos grupos de crías estuvieron expuestos a la ROT durante el mismo tiempo (la diferencia entre estos estriba en las crías que permanecieron sin exposición durante 30 días), el daño aparentemente mayor que la ROT provocó sobre las neuronas de las crías de 60 DPN no se correlacionó con los resultados de la coordinación motora, ya que no hubo diferencias significativas entre los animales probados a los 30 días y los probados a los 60 días de edad, en el tiempo de ejecución para trepar por la viga inclinada (figura 4).

Durante la etapa fetal el cerebro es sensible a los daños que pueden ocasionarle diversos factores externos; durante este periodo, la protección que ofrece la placenta es limitada, sobre todo contra aquellas sustancias que son altamente liposolubles como la ROT. Además, en los fetos la barrera hematoencefálica no se encuentra completamente desarrollada pues ésta termina su desarrollo después del nacimiento, ${ }^{22}$ y debido a que el cerebro continúa su desarrollo durante la etapa posnatal, el periodo de vulnerabilidad se prolonga. Se ha reportado que ratones recién nacidos expuestos a dosis bajas de agentes neurotóxicos, como el insecticida paraquat, no muestran efectos inmediatos; sin embargo en la edad adulta presentan cambios conductuales y deficiencias en el aprendizaje, lo que se ha interpretado como una consecuencia de los daños cerebrales provocados por la exposición temprana a esta substancia. ${ }^{11,23}$

Barlow et al. ${ }^{11}$ mostraron que la presencia de pesticidas durante el periodo prenatal y perinatal causa la reducción del número de neuronas dopaminérgicas e incrementa la susceptibilidad de estas neuronas a la degeneración por daños subsecuentes ocasionados por otros factores ambientales o por la edad. ${ }^{24-26}$ Nuestros resultados parecen apoyar sus hipótesis.

Se ha sugerido que los niños accidentalmente expuestos durante el desarrollo fetal a pesticidas tienen mayor riesgo de desarrollar trastorno de déficit de atención e hiperactividad (TDAH), sobre todo en casos donde la exposición es mediante alimentos tratados con pesticidas. ${ }^{27,28} \mathrm{El}$ TDAH está estrechamente relacionado con trastornos en el funcionamiento del sistema dopaminérgico. Warton sugiere que los niños que lo padecen pueden tener alterada la liberación de dopamina en las terminales nigroestriatales. ${ }^{29}$ Nuestro estudio sugiere que la exposición a ROT por vía materna podría tener alguna relación causal con sus observaciones.

Adicionalmente, se ha documentado que la exposición a pesticidas, entre los cuales se encuentra la ROT, aumenta la incidencia de la enfermedad de Parkinson en los indivi- 
duos expuestos., ${ }^{3,40}$ Por otra parte, Whatley ha propuesto un mecanismo fisiopatológico indirecto para el desarrollo de esquizofrenia, derivado de que la ROT puede afectar la expresión de genes mitocondriales en las neuronas dopaminérgicas, y esto, a su vez, podría facilitar el desarrollo de dicha enfermedad. ${ }^{31}$ Más aún, entre los efectos de la ROT está la disminución de serotonina en el hipocampo, que facilita conductas relacionadas con la depresión, que también han sido exploradas en modelos animales. ${ }^{32}$

En resumen, la ROT es capaz de inducir daño neuronal desde antes del nacimiento; los individuos expuestos durante su desarrollo podrían tener un número menor de neuronas dopaminérgicas desde etapas tempranas de la vida, lo que los haría más vulnerables a otros factores nocivos, aumentando el riesgo de desarrollar en edades tempranas trastornos relacionados con disfunciones del sistema dopaminérgico como el TDAH, o alteraciones tardías como la enfermedad de Parkinson, sin descartar otros tipos de trastornos funcionales o psiquiátricos.

Hasta donde sabemos, en México se conoce muy poco sobre la asociación entre xenobióticos ambientales y trastornos neurológicos en individuos expuestos durante etapas tempranas de la vida. Se supone que en México hay aproximadamente 1.5 millones de niños menores de 18 años que padecen de TDAH, y la mayoría no han sido diagnosticados. ${ }^{33}$ Existe la posibilidad de que un número importante de individuos se encuentre afectado debido a la exposición in utero a tóxicos ambientales. ${ }^{13}$

\section{AGRADECIMIENTOS}

A Gabriel Pérez Soto por su asistencia técnica. Este proyecto fue financiado por el proyecto 102314 del CONACyT.

\section{REFERENCIAS}

1. Kilbourn MR, Charalambous A, Frey KA, Sherman P et al. Intrastriatal neurotoxin injections reduce in vitro and in vivo binding of radiolabeled rotenoids to mitochondrial complex I. J Cereb Blood Flow Metab 1997;17(3):265-272.

2. Betarbet R, Sherer TB, MacKenzie G, Garcia-Osuna M et al. Chronic systemic pesticide exposure reproduces features of Parkinson's disease. Nat Neurosci 2000;3(12):1301-1306.

3. Dhillon AS, Tarbutton GL, Levin JL, Plotkin GM et al. Pesticide/environmental exposures and Parkinson's disease in East Texas. J Agromedicine 2008;13(1):37-48.

4. Vanacore N, Nappo A, Gentile M, Brustolin A et al. Evaluation of risk of Parkinson's disease in a cohort of licensed pesticide users. Neurol Sci 2002;23(2):S119-5120.

5. Moretto A, Colosio C. Biochemical and toxicological evidence of neurological effects of pesticides: The example of Parkinson's disease. Neurotoxicology 2011;32(4):383-391.

6. Wang A, Costello S, Cockburn M, Zhang X et al. Parkinson's disease risk from ambient exposure to pesticides. Eur J Epidemiol 2011;26(7):547-555.

7. Sherer TB, Kim JH, Betarbet R, Greenamyre JT. Subcutaneous rotenone exposure causes highly selective dopaminergic degeneration and alpha-synuclein aggregation. Exp Neurol 2003;179(1):9-16.
8. Schapira AH, Cooper JM, Dexter D, Jenner P et al. Mitochondrial complex I deficiency in Parkinson's disease. Lancet 1989;1(8649):1269.

9. Paxinos G, Watson Ch. The rat brain in stereotaxic coordinates. San Diego: Academic Press; 1997.

10. Grandjean P, Landrigan PJ. Developmental neurotoxicity of industrial chemicals. Lancet 2006;368(9553):2167-2178.

11. Brown TP, Rumsby PC, Capleton AC, Rushton $L$ et al. Pesticides and Parkinson's disease- is there a link? Environ Health Perspect 2006;114(2):156-164.

12. Trash B, Uthayathas S, Karuppagounder SS, Suppiramaniam V et al. Paraquat and maneb induced neurotoxicity. Proc West Pharmacol Soc 2007;50:31-42.

13. Barlow BK, Cory-Slechta DA, Richfield EK, Thiruchelvam M. The gestational environment and Parkinson's disease: evidence for neurodevelopmental origins of a neurodegenerative disorder. Reprod Toxicol 2007;23(3):457-470.

14. Wigle DT, Arbuckle TE, Walker M, Wade MG et al. Enviromental hazards: Evidence for efectos on child health. J Toxicol Envir Health Part B 2007;10:3-39.

15. Alam M, Schmidt WJ. Rotenone destroys dopaminergic neurons and induces parkinsonian symptoms in rats. Behav Brain Res 2002;136(1):317-324.

16. Fleming SM, Zhu C, Fernagut PO, Mehta A et al. Behavioral and immunohistochemical effects of chronic intravenous and subcutaneous infusions of varying doses of rotenone. Exp Neurol 2004;187(2):418-429.

17. Luo C, Rajput AH, Akhtar S, Rajput A. Alpha-synuclein and tyrosine hydroxylase expression in acute rotenone toxicity. Int J Mol Med 2007;19(3):517-521.

18. Cannon JR, Tapias V, Na HM, Honick AS et al. A highly reproducible rotenone model of Parkinson's disease. Neurobiol Dis 2009;34(2):279-290.

19. Thiruchelvam M, Richfield EK, Goodman BM, Baggs RB et al. Developmental exposure to the pesticides paraquat and maneb and the Parkinson's disease phenotype. Neurotoxicology 2002;23(4-5):621-633.

20. Cory-Slechta DA, Thiruchelvam M, Barlow BK, Richfield EK. Developmental pesticide models of the Parkinson disease phenotype. Environ Health Perspect 2005;113(9):1263-1270.

21. Cicchetti D, Walker E. Neurodevelopmental Mechanisms in Psychopathology. Cambridge University Press; 2003.

22. Julvez J, Grandjean P. Neurodevelopmental toxicity risks due to occupational exposure to industrial chemicals during pregnancy. Ind Health $2009 ; 47(5): 459-468$.

23. Eriksson P. Developmental neurotoxicity of environmental agents in the neonate. Neurotoxicology 1997;18(3):719-726.

24. Heindel JJ. The fetal basis of adult disease: Role of environmental exposures-introduction. Birth Defects Res A Clin Mol Teratol 2005;73(3):131-132.

25. Landrigan PJ, Sonawane B, Butler RN, Trasande L et al. Early environmental origins of neurodegenerative disease in later life. Environ Health Perspect 2005;113(9):1230-1233.

26. Richardson JR, Caudle WM, Wang M, Dean ED et al. Developmental exposure to the pesticide dieldrin alters the dopamine system and increases neurotoxicity in an animal model of Parkinson's disease. FASEB J 2006;20(10):1695-1697.

27. Mehler-Wex C, Riederer P, Gerlach M. Dopaminergic dysbalance in distinct basal ganglia neurocircuits: implications for the pathophysiology of Parkinson's disease, schizophrenia and attention deficit hyperactivity disorder. Neurotox Res 2006;10(3-4):167-179.

28. Kuehn BM. Increased risk of ADHD associated with early exposure to pesticides, PCBs. JAMA 2010;304(1):27-28.

29. Warton FL, Howells FM, Russell VA. Increased glutamate-stimulated release of dopamine in substantia nigra of a rat model for attentiondeficit/hyperactivity disorder-lack of effect of methylphenidate. Metab Brain Dis 2009;24(4):599-613.

30. Wirdefeldt K, Adami HO, Cole P, Trichopoulos D et al. Epidemiology and etiology of Parkinson's disease: a review of the evidence. Eur J Epidemiol 2011;26 (Supl 1):S1-58. 
31. Whatley SA, Curti D, Marchbanks RM. Mitochondrial involvement in schizophrenia and other functional psychoses. Neurochem Res 1996;21(9):995-1004.

32. Santiago RM, Barbieiro J, Lima MM, Dombrowski PA et al. Depressive-like behaviors alterations induced by intranigral MPTP, 6-OHDA, LPS and rotenone models of Parkinson's disease are predominantly associated with serotonin and dopamine. Prog Neuropsychopharmacol Biol Psychiatry 2010;34(6):1104-1114.

33. Dirección General de Servicios de Salud Mental. Programa de Acciones en Salud Mental. Análisis de la Problemática de la Salud Mental en México. Diagnostico Epidemiológico. Cap. III, Año 2002, págs. 4357 (http://sersame.salud.gob.mx/pdf/pasm_cap3.pdf).

Artículo sin conflicto de intereses 